\title{
The ethics of complementary therapy research recruitment: a case study
}

We suggest that physicians responsible for the clinical care of their patients have a moral and ethical duty to thoughtfully consider recruitment to appropriately approved, funded, and peer reviewed research that may possibly help the people for whom they are responsible. This facilitates the sustained development of evidence-based medicine while recognising the clinical needs of their patients and their rights to informed consent. We do not believe that physicians have the right to fail to inform or exclude patients from such studies based on their personal beliefs. We present a case study that illustrates this issue within the context of researching spiritual healing and cancer.

\section{Introduction.}

CAM use has increased considerably in the Western industrialised nations over the last 25 years with around $83 \%$ of cancer patients using it in some form, ${ }^{1}$ almost invariably in conjunction with conventional medicine, to ameliorate the side effects of treatments. ${ }^{2}$ About $40 \%$ of CAM users consulted concurrently with their orthodox physician about the same problem and self manage their CAM and conventional medical treatments, ${ }^{3,4}$ an approach which has generated considerable debate within the context of oncology.

There are currently about 14000 registered spiritual healers in the UK. ${ }^{5}$ Spiritual healing is an ancient approach to illness which acknowledges the spiritual dimension of human existence. It does not refer in any way to religion or a specific religious belief system. ${ }^{6}$ There is some preliminary evidence to suggest it can create profound relaxation and pain relief $^{7-11}$ which may also improve depression ${ }^{7,8,11-13}$ and anxiety ${ }^{8,10-12}$ as well as encourage sleep $p^{9,13}$ and engender inner peace, ${ }^{7,14}$ thus potentially improving wellbeing and quality of life. . $7,7,8,10,12,13$
Healing is used as a complementary therapy during a 'time of need'. Patients appear to use spiritual practices for their additive effect and do not seem to be tempted to abandon conventional treatment while using healing. ${ }^{1,4}$ Healers do not diagnose or prescribe; they should not claim any specific 'medical' effects for healing nor should they have any attachment to a particular outcome. While the spiritual aspect of wellbeing is a much debated topic, individuals with greater spiritual wellbeing appear to have lower levels of anxiety. ${ }^{15}$ Therefore understanding these aspects of a lifethreatening illness may help patients to adjust to their diagnosis of cancer, identifying the support they need to help them overcome their fear as well as enabling them to find ' personal meaning' in their life. ${ }^{16}$

Discussions about spiritual healing are rarely met dispassionately as the debate appears to evoke emotions which range from support, through indifference to vehement disapproval, perhaps because of its (incorrectly) perceived religious context. It is probably the oldest documented medical intervention which, while currently broadly available and used widely, is a neglected area of research. Rigorous research into spiritual healing poses many methodological challenges if we are to thoughtfully address the issues of efficacy and safety.

\section{Our research proposal}

With these provisos in mind we developed an initial qualitative protocol using Unitary Appreciative Inquiry ${ }^{17}$ to begin to evaluate the potential benefits of spiritual healing for people with cancer who were also suffering well-documented adverse drug reactions from life-saving medications. We wished to identify any patient-centred changes that occur as a consequence of healing and planned subsequently to develop appropriate methodology for a randomised controlled trial, bearing in mind the MRC complex interventions guidelines. We are concerned about the use of placebos in trials of spiritual healing because we have no understanding of how healing may work and are therefore at a complete loss to know how to design an appropriate and valid placebo. The use of a 'sham' healer as placebo may not be reasonable or possible as a person pretending to heal might actually be able to heal, unbeknown to themselves, the patients, or the researchers. Crossover trials are also not appropriate because unlike pharmaceutical products, we cannot predict the dose response or duration of effect for healing without having some insight as to the mechanisms involved. It may be that the therapeutic mechanisms involved in healing are related to patient expectation and an enhanced placebo effect, thus complicating the design of randomised clinical trials which implies a specific treatment effect as a consequence of spiritual healing. ${ }^{18}$

\section{The study}

We chose to study patients on established cancer treatment because they have a well-documented disease course. Research has indicated that a substantial proportion of patients find it difficult to maintain their long-term medication regime because of adverse drug reactions and are tempted to indulge in potentially dangerous 'drug holidays'19 that may reduce life expectancy. We hypothesised that some of the documented effects of spiritual healing, might assist these patients in maintaining their essential conventional adjuvant treatment regime, by managing these adverse reactions. All patients in this study were advised to continue with their orthodox medicines.

Oncologists were involved at all stages 
of the trial design from the outset. The initial protocol was developed in discussion with a clinician who also had research skills and then reviewed and accepted by the local Cancer Care Directorate peer review committee. It was then approved for the purposes of research governance after consideration by the local NHS Research and Development unit. The study subsequently received ethical approval and after further discussion and review was located at a dedicated research facility within a hospital trust. We sought funding for the study from a healing charity and this was granted.

For the initial qualitative phase of the study we planned to recruit up to 20 patients through the local clinic. It was agreed at the review and ethics stages that the oncologists would make available patient information packs to suitable patients. Patients could then contact the researcher if they wanted to participate in the study or required further information. The oncologists were not required to explain the study or actively recruit patients.

A senior consultant clinician refused to allow any of the patients in his care to receive the study information packs as he personally deemed spiritual healing to be 'hocus pocus'. The nurses and less senior members in the clinical team were initially enthusiastic and agreed to hand out the patient information packs, but this hostile reaction permeated through the whole clinical team and informal observations indicate that there was both overt and covert refusal by the team to inform patients about the study. Thus the agreed route of recruitment became impossible and patients who might have benefited from the study treatment were actively denied information and therefore denied the choice of whether to participate.

\section{Conclusions}

Pervious editorials have frequently called for more research into CAM and we would entirely agree with this sentiment. Within a research context it is acceptable for a physician to feel personally unable to support a specific research project, but in such circumstances, the clinician in question would appear to have an obligation to inform patients of the study and refer potential patients to a more cooperative colleague. This should be easy to achieve in an environment where patients do not necessarily see the same doctor or nurse on each occasion. It is however, unacceptable in our view, to obstruct a legitimately approved and funded study thus preventing informed patient choice and intentionally thwarting a research project based on individual personal opinion.

In order to recruit onto our study we approached the local press and TV with information about the study so potential participants could contact us through the media. They could then receive information packs and if they chose to participate and fulfilled the inclusion criteria, we sought permission directly from their GP or consultant prior to their participation in the study.

Our success in overcoming this obstruction to recruitment does not properly address the issues raised by the resistance we experienced. This funded study was almost thwarted and there appeared to be no mechanism for redress within the existing system. Future CAM studies may also encounter similar difficulties, in spite of support from some members of the clinical team, and have to seek new and unique ways to circumvent such barriers to research or run the risk of wasting public or charitable money given to support research. Surely orthodox medicine, which frequently cites lack of evidence for CAM in general as a reason for their reticence to use or recommend it, ${ }^{20}$ has a duty to support research and facilitate recruitment onto thoughtfully developed, ethically approved studies. Such opposition to research within spiritual healing serves no one well, least of all our needy patients.

\section{Fiona Barlow and George Lewith}

\section{REFERENCES}

1. Richardson M, Sanders T, Palmer J, et al. Complementary/alternative medicine use in comprehensive cancer center and the implications for oncology. J Clin Oncol 2000; 18(13):2505-2514.

2. Humpel N, Jones S. Gaining insight into the what, why and where of complementary and alternative medicine use by cancer patients and survivors. Eur J Cancer Care 2006; 15: 362-368.

3. Shmueli A, Shuval J. Complementary and alternative medicine: beyond users and nonusers. Complement
Ther Med 2006; 14: 261-267.

4. Henderson J, Donatelle R. Complementary and alternative medicine use by women after completion of allopathic treatment for breast cancer. Altern Ther Health Med 2004; 10(1): 52-57.

5. Ernst E. Spiritual healing: more than meets the eye. Journal of Pain and Symptom Management 2006; 32(5): 393-395.

6. Geddes \& Grosset. Guide to natural healing. Geddes \& Grosset: Scotland, 1997.

7. Pohl G, Seemann H, Zojer N, et al. 'Laying on of hands' improves well-being in patients with advanced cancer. Support Care Cancer 2007; 15: 143-151.

8. Schmehr R. Enhancing the treatment of HIV/AIDS with Reiki training and treatment. Altern Ther Health Med 2003; 9(2): 120-121.

9. Sundblom D, Haikonen S, Niemi-Pynttari J, Tigerstedt I. Effect of spiritual healing on chronic idiopathic pain: a medical and psychological study. Clin J Pain 1994; 10(4): 296-302.

10. Tsang K, Carlson LE, Olson K. Pilot crossover trial of Reiki versus rest for treating cancer-related fatigue. Integ Cancer Ther 2007; 6(1): 25-35.

11. Miles P. Preliminary report on the use of Reiki for HIVrelated pain and anxiety. Altern Ther Health Med 2003; $9(2): 36$.

12. Dixon M. Does 'healing' benefit patients with chronic symptoms? A quasi-randomized trial in general practice. J R Soc Med 1998; 91:183-188.

13. Vaghela C, Robinson N, Gore J, et al. Evaluating healing for cancer in a community setting from the perspective of clients and healers: a pilot study. Complement Ther Clin Pract 2007; 13(4): 240-249.

14. Brown CK. Is spiritual healing a valid and effective therapy? J R Soc Med 1995; 88(12): 722.

15. Kaczorowski J. Spiritual well-being and anxiety in adults diagnosed with cancer. Hosp J 1989; 5(34):105-116.

16. Coward DD, Kahn DL. Resolution of spiritual disequilibrium by women newly diagnosed with breast cancer. Oncol Nurs Forum 2004; 31(2): E24-E31.

17. Cowling WR. Pattern, participation, praxis, and power in unitary appreciative inquiry. ANS Adv Nurs Sci 2004; 27(3):202-214.

18. Walach $\mathrm{H}$, Bosch $\mathrm{H}$, Lewith $\mathrm{G}$, et al. Effectiveness of distant healing for patients with chronic fatigue syndrome: a randomized controlled partially blind tria (EUHEALS). Psychotherapy \& Psychosomatics 2008; 77(3):158-166.

19. Fallowfield L, Atkins L, Catt S, et al. Patients' preference for administration of endocrine treatments by injection or tablets: results from a study of women with breast cancer. Annals of Oncology 2005; Advance Access October 2005; 2006; 17: 205-210.

20. Baum M, Ashcroft F, Berry C, et al. Doctors' campaign against alternative therapies.

http://www.timesonline.co.uk/tol/news/uk/health/articl e723787.ece (accessed 10 Mar 2009).

DOI: 10.3399/bjgp09X420509 